tige Technowissenschaft wird gerade im Anwendungsteil nicht konsequent eingelöst. Fraglich - das allerdings gilt es nicht explizit für dieses Buch zu diskutieren, sondern ist zumindest ein weiteres Charakteristikum der sich um die Synthetische Biologie rankenden Auseinandersetzungen - ist, wen genau die Konzeptionalisten eines derart facettenreichen und fachspezifischen Diskurses vor Augen haben. Die vielbeschworene allgemeine Öffentlichkeit mag es nicht sein.

$《 》$

\section{Von der Wissenschaftsforschung zur Wissenschaftspolitik}

E. Grande, D. Jansen, O. Jarren, A. Rip,
U. Schimank, P. Weingart (Hg.): Neue
Governance der Wissenschaft. Reorgani-
sation - externe Anforderungen - Mediali-
sierung. Bielefeld: transcript 2013, 374 S.,
ISBN 978-3-8376-2272-0, Euro 32,80

Rezension von David Kaldewey, Abteilung Wissenschaftsforschung, Universität Bonn

\section{Wissenschaft und Politik}

Zur Charakterisierung des Verhältnisses von Wissenschaft und Politik werden seit Harvey Brooks (1968) zwei Einflussrichtungen unterschieden: Auf der einen Seite geht es um die Verwendung wissenschaftlichen Wissens in politischen Entscheidungsprozessen, vermittelt etwa durch wissenschaftliche Politikberatung (science for policy), auf der anderen Seite um die politische Aufgabe der Aufrechterhaltung eines funktionierenden Wissenschaftssystems, etwa durch Finanzierung von Institutionen oder Forschungsprogrammen (policy for science). Dass diese Unterscheidung nicht immer scharf zu ziehen ist, hat Brooks nie bestritten, und lässt sich auch an einer Beobachtung von Rudolf Stichweh (2013) illustrieren, der zufolge Wissenschaftspolitik im zweiten Sinne häufig von Wissenschaftlern betrieben wird, die dauerhaft oder temporär die Rolle wissenschaftlicher Politikberater übernehmen, und in dieser neuen Rolle die Wissenschaft politisch zu steuern suchen. Ein weiterer Sonderfall liegt vor, wenn die politikberatende Rolle nicht von ,normalen' Wissenschaftlern übernommen wird, die im Verlauf ihrer Karriere mit Seitenwechseln experimentieren, sondern von Wissenschaftsforschern, die sich thematisch auf die Wissenschaft als soziales System und auf deren Verhältnis zur gesellschaftlichen Umwelt spezialisiert haben. Tatsächlich zeichnen sich insbesondere die Science and Technology Studies (STS) durch einen entsprechenden Trend zur Politisierung aus: Steve Fuller (2007) etwa charakterisiert die STS als „political player“ und Sergio Sismondo (2008) skizziert in diesem Sinne ein ,engaged program“, das sich neben der epistemischen Durchdringung des Gegenstandes auch politischen Werten verpflichtet weiß.

Diese für die Identitätsarbeit der STS konstitutive Politisierung ist eine hilfreiche Folie zur Interpretation der in den letzten Jahren auch in Deutschland engagierter geführten Diskussion über das Verhältnis von Wissenschaftsforschung und Politik. Der von Edgar Grande et al. herausgegebene Sammelband „Neue Governance der Wissenschaft" lädt in diesem Zusammenhang dazu ein, anhand einer Reihe von Forschungsprojekten zu eruieren, wie die deutsche Wissenschaftsforschung - die sich bislang im Vergleich zu den dominanten Strömungen der STS durch ein „distanzierteres“ Verhältnis zu ihrem Forschungsgegenstand ausgezeichnet hatte - mit der Frage der politischen Relevanz umgeht.

\section{Die Rolle der BMBF-Programmförderung}

Die in dem Band präsentierten Projekte sind im Rahmen einer breit angelegten Förderinitiative des Bundesministeriums für Bildung und Forschung (BMBF 2008) durchgeführt worden. Hintergrund ist ein bereits 2001 aufgelegtes Programm, das darauf zielte, die in Deutschland - zumal im Vergleich mit anderen europäischen Ländern - nur schwach institutionalisierte Wissenschaftsforschung durch gezielte Schwerpunktbildung besser aufzustellen. Lanciert wurde daraufhin die zweiphasige, mit einem Volumen von rund 15 Mio. Euro ausgestatte Förderinitiative „Forschung zum Verhältnis von Wissenschaft, Politik und Gesellschaft“. In der 
ersten Phase (2003-2008) standen unter dem Titel „Wissen für Entscheidungsprozesse“ Fragen der Wissensproduktion und der wissenschaftlichen Politikberatung im Vordergrund (s. dazu die Dokumentation bei Mayntz et al. 2008), in der zweiten Phase (2009-2013) dagegen strukturelle Aspekte des Wissenschaftssystems, die nun unter der Überschrift „Neue Governance der Wissenschaft" publiziert sind. In der Trennung der beiden Förderphasen klingt die Unterscheidung von science for policy und policy for science an, die allerdings nicht explizit verwendet wird, vermutlich weil gerade die zweite Phase darauf zielte, die mittlerweile etwas verstaubte Begrifflichkeit durch die zeitgemäßere Governance-Semantik zu ersetzen. Vergleicht man den Buchtitel mit Brooks' Klassiker - „The Government of Science" (1968) - dann liegt die doppelte Abgrenzung auf der Hand: Es geht um „Governance“, nicht um „Government“, und dazu auch um eine „neue“" Form derselben.

Im Vorwort des Bandes findet sich neben der Erläuterung des Entstehungskontextes auch eine kurze Bilanz aus Sicht des Projektträgers. Hier werden zwei Ziele hervorgehoben, die aus förderpolitischer Sicht mit dem Programm verbunden waren: ,zum einen die Produktion von wissenschaftspolitisch relevanten Erkenntnissen und Empfehlungen, zum anderen eine Stärkung der Wissenschaftsforschung in Deutschland" (S. 13). Der mit dieser doppelten Zielsetzung markierten Herausforderung stellen sich die Herausgeber auf verschiedene Weise. Erstens definieren sie den Ausgangspunkt des Bandes mit Bezug auf das „Spannungsverhältnis von wissenschaftlicher Autonomie und staatlicher Steuerung" (S. 15) und betonen damit, dass die Wissenschaftsforschung sowohl die Eigengesetzlichkeiten wie die gesellschaftlichen Kontextbedingungen der Wissenschaft im Blick behalten muss. Mit der Forderung nach der Verbindung dieser Perspektiven stellen sich die Herausgeber implizit in die Tradition der Internalismus/Externalismus-Debatte (Shapin 1992). Zweitens heben sie hervor, dass die Wissenschaftspolitik nicht einfach für einen Pol dieser Differenz steht, sondern sich vielmehr selbst in diesem Spannungsfeld bewegt: Die Bewahrung der „Autonomie der Wissenschaft“ ist nicht weniger eine politische (letztlich grundge- setzlich verankerte) Zielsetzung als die thematische „Prioritätensetzung“ oder die vor dem Hintergrund anderer gesellschaftlicher Werte notwendige „Regulierung von Forschung" (S. 17). Die Lösung im Umgang mit dieser Spannung, so die Diagnose, lautete lange Zeit ,gesteuerte Autonomie“. Angesichts vielfältiger Transformationen und komplexer werdenden Regelungsmechanismen in allen gesellschaftlichen Bereichen sei dieses Modell jedoch an seine Grenzen gekommen. An seine Stelle trete eine neue „Governance of science“, deren Konturen sich bereits andeuten, deren sozialwissenschaftliche Durchdringung aber noch ausstehe (S. 19).

\section{Inhaltsüberblick}

Gegliedert ist der Band, wie im Untertitel angedeutet (,Reorganisation - externe Anforderungen - Medialisierung"), in drei Teile, die in ihrer thematischen Ausrichtung schon je für sich genug Stoff für ein eigenständiges Buch geliefert hätten. Im ersten Teil geht es um den Wandel der Governance-Regime von Hochschulen. Hier ist der Bezug zum Überthema am deutlichsten, was daran liegt, dass das Governance-Konzept im Kontext der Hochschulforschung leichter operationalisierbar ist als in anderen Bereichen der Wissenschaftsforschung: Es ist hier unmittelbar verknüpft mit den staatlichen Reformbestrebungen der letzten zwei Jahrzehnte und mit der Frage nach den funktionalen und dysfunktionalen Effekten des New Public Management (NPM). Neben einem umfassenden Überblick über die neuen Steuerungsinstrumente und deren Umsetzungsstand an deutschen Universitäten (Bogumil et al.) finden sich weitere Studien zu den Effekten der gegenwärtigen Evaluationskultur (Gläser/von Stuckrad), zur Effektivität der Leistungsorientierten Mittelvergabe als einem paradigmatischen NPM-Instrument (Krempkow/ Landrock) und zur Frage, ob die Wissenschaftspolitik neben der Spitzenförderung im Rahmen der Exzellenzinitiative nicht auch Programme auflegen sollte, die gezielter in die Breite wirken (Grözinger/Fromm). Auch wenn sich diese Beiträge weitgehend der Hochschulforschung zuordnen lassen, betonen die Herausgeber die Bedeutung solcher Studien auch für die Wissen- 
schaftsforschung: Denn bei der Hochschulpolitik bzw. den Governance-Reformen, so die starke, allerdings nicht systematisch begründete These, handele es sich um den „Hauptkanal“, über den der außerwissenschaftliche Kontext an die Wissenschaft herangetragen werde (S. 30).

Im zweiten Teil geht es um „weitere“, d. h. über andere Kanäle vermittelte oder angestoßene „Irritationen der Autonomieansprüche von Wissenschaft" (S. 30). Thematisiert werden insbesondere intersystemische Beziehungen zu anderen gesellschaftlichen Teilsystemen. Die Beiträge widmen sich allerdings nicht den abstrakten "Großtrends“ (wie etwa der Ökonomisierung der Wissenschaft), sondern setzen eine Stufe darunter an: Im Sinne des durch die Förderinitiative vorgegebenen konzeptuellen Rahmens soll es auch hier um die ,gegenstandsbezogenen Stellschrauben“ gehen, „die von relevanten Akteuren zu Gestaltungszwecken genutzt werden können“" (S. 31). Versammelt sind hier Beiträge zur Identitätsarbeit von Ressortforschungseinrichtungen im Spannungsfeld von akademischen und politischen Anforderungen (Bach et al.), zur Einbettung der Hochschulen in ihr regionales Umfeld und die damit einhergehenden Potenziale strategischer Netzwerkbildung (Koschatzky et al.), zum sich transformierenden Patentsystem und zum Problem der „Governance von Innovationen“ (Böschen et al.), zur Möglichkeit „uneingeladener" Partizipation der Zivilgesellschaft in Forschung und Wissenschaftspolitik (Wehling/ Viehöver) sowie zur Frage, wie sich die neue Governance der Wissenschaft aus einer GenderPerspektive darstellt (Riegraf/Weber).

Der dritte Teil konzentriert sich auf den in der gegenwärtigen Diskussion als besonders prominent wahrgenommen Kontextfaktor der Medialisierung. Der Bezug zur Governance-Problematik liegt hier nicht immer auf der Hand, wird aber durch die These plausibilisiert, dass Medialisierung ,neue Plattformen“ für die wechselseitige Beobachtung von Wissenschaft und Politik schafft; eine Funktion, die von den Herausgebern als ,elementarer Governance-Mechanismus" beschrieben wird (S. 36). Konkret untersucht wird das sich wandelnde Verhältnis der Hochschulen zur Öffentlichkeit (Marcinkowski et al.), die Rolle der Medien für die Forschungspolitik (Blö- baum et al.), die indirekt über die Medien vermittelte Kommunikation zwischen Wissenschaft und Politik am Beispiel der Neurowissenschaften (Peters et al.) sowie der Zusammenhang von Publikationspraktiken und Medialisierungstendenzen in verschiedenen Fachkulturen (Franzen/ Rödder). Bei allen Beiträgen fällt auf, dass die etwa im Rahmen von Befragungen immer wieder geäußerte Vermutung, Wissenschaftspolitiker und Wissenschaftsförderer orientierten sich an der „öffentlichen Sichtbarkeit von Forschern, Projekten und Ergebnissen“" (S. 332), empirisch ungedeckt bleibt: Es hätte den Rahmen dieser Studien gesprengt, einen Seitenwechsel von der Wissenschaft zur Wissenschaftspolitik zu vollziehen, der methodisch ja darauf hinauslaufen müsste, sich nicht mehr nur der Wissenschaftskommunikation, sondern - mit allen Mitteln der empirischen Sozialforschung - dem Feld der Wissenschaftspolitik selbst zu widmen.

\section{Die Ausblendung der Wissenschaftspolitik}

Zusammenfassend lässt sich festhalten, dass sich der Band in empirischer Hinsicht wissenschaftlichen Organisationen, insbesondere Hochschulen, sowie wissenschaftlichen Disziplinen und Fachkulturen widmet. Das Verhältnis von Wissenschaft und Politik wird entsprechend primär aus der Binnenperspektive der Wissenschaft analysiert, die Wissenschaftspolitik selbst - sei es in Form von Förderorganisationen, staatlichen Ministerien oder sonstigen Akteuren - ist nicht Gegenstand empirischer Beobachtung. Sie ist Adressat (insbesondere für Handlungsvorschläge, die in fast jedem Beitrag formuliert werden) und erscheint im empirischen Material immer wieder als zentrale Referenz. Wie diese black box funktioniert, bleibt jedoch weitgehend der Spekulation überlassen, die Autoren der verschiedenen Studien sind hier sehr zurückhaltend und beschränken sich auf ein Referieren von Meinungen der jeweils untersuchten Akteure. Vor diesem Hintergrund wird deutlich, dass eine Soziologie der Wissenschaftspolitik, oder vielleicht spezifischer, eine Wissenssoziologie der Wissenschaftspolitik, ein Desiderat ist. Offene Fragen sind beispielsweise, welches Wissen der wissenschaftspolitischen Praxis zugrunde liegt, inwieweit dieses Wissen 
selbst wissenschaftlichen Kriterien genügt, und ob das Wissen der Wissenschaftsforschung in diesem Zusammenhang überhaupt eine relevante Rolle spielt. Weiter wäre dann die Perspektive wieder zu wechseln, um zu untersuchen, wie das der Wissenschaftspolitik implizit oder explizit zugrundeliegende Wissen auf den regulierten Gegenstand, die Wissenschaft, zurückwirkt.

Die Ausblendung der Wissenschaftspolitik als empirischer Gegenstand vis-à-vis der Wissenschaft geht einher mit der Ausblendung der „Politisierung“ der Wissenschaft als einem klassischen Thema der älteren Forschungsliteratur. Nur in einem einzigen Beitrag taucht der Begriff der Politisierung überhaupt auf. Marcinkowski et al. interpretieren die von ihnen untersuchten Medialisierungsprozesse als eine ,indirekte Politisierung“ bzw. als ein internalisiertes ,wissenschaftspolitisches Zustimmungsmanagement" (S. 257), mit dem die Hochschulen sich gewissermaßen antizipativ den vermuteten politischen Erwartungen und Umweltanforderungen anpassen. Hier drängt sich die Vermutung auf, dass in einem Förderkontext, der die wissenschaftspolitische Relevanz der Wissenschaftsforschung fokussiert, die Politisierung selbst nur indirekt, in diesem Fall durch die Kopplung mit dem Phänomen der Medialisierung, thematisiert werden kann. Tatsächlich scheinen die Weichen für die weitgehende Ausblendung von Phänomenen der Politisierung schon mit der Ausschreibung der Förderinitiative gestellt worden zu sein. Unter der Überschrift „Gegenstand der Förderung“ wurden vier Faktoren der gegenwärtigen Transformationsprozesse genannt, die es zu erforschen gelte: Ökonomisierung, Instrumentalisierung, Medialisierung und Transnationalisierung. In der Logik der Förderinitiative, so kann man vermuten, wäre es problematisch, wenn die Politisierung der Wissenschaft als ein externer Faktor unter diesen anderen erschiene, denn die Aufgabe, die der Politik implizit zugewiesen wird, ist es ja gerade, an Lösungen für den Umgang mit diesen anderen Faktoren mitzuwirken. Zwischen den Zeilen klingt auch die aus den STS bekannte Idee an, dass die Wissenschaft einer Politisierung bedarf, um besser gewappnet zu sein gegen die genannten Trends etwa der Ökonomisierung oder der Medialisierung. Diese These gilt es nicht per se $\mathrm{zu}$ verwerfen, wohl aber wäre für die Zukunft genauer nachzufragen, welche funktionalen und dysfunktionalen Aspekte mit den verschiedenen, noch genauer zu unterscheidenden Formen der Politisierung der Wissenschaft einhergehen und ob im Falle von Dysfunktionalitäten die Politik die richtige Adresse ist, um eben diese Effekte zu korrigieren.

\section{Literatur}

$B M B F$ - Bundesministerium für Bildung und Forschung, 2008: Bekanntmachung der Förderrichtlinien „Neue Governance der Wissenschaft - Forschung zum Verhältnis von Wissenschaft, Politik und Gesellschaft"; http://www.bmbf.de/foerderungen/12150.php (download 27.5.15)

Brooks, H., 1968: The Government of Science. Cambridge, MA

Fuller, S., 2007: New Frontiers in Science and Technology Studies. Cambridge/Malden

Mayntz, R.; Neidhardt, F.; Weingart, P. et al. (Hg.), 2008: Wissensproduktion und Wissenstransfer. Wissen im Spannungsfeld von Wissenschaft, Politik und Öffentlichkeit. Bielefeld

Shapin, S., 1992: Discipline and Bounding. The History and Sociology of Science as Seen through the Externalism-Internalism Debate. In: History of Science 30 (1992), S. 333-369

Sismondo, S., 2008: Science and Technology Studies and an Engaged Program. In: Hackett, E.J.; Amsterdamska, O.; Lynch M.E. et al. (Hg.): The Handbook of Science and Technology Studies. Cambridge, S. 13-31

Stichweh, R., 2013: Differenzierung von Wissenschaft und Politik. Wissenschaftspolitik im 19. und 20. Jahrhundert. In: Stichweh, R. (Hg.): Wissenschaft, Universität, Professionen. Soziologische Analysen. Bielefeld, S. 135-150 